\title{
Incidência e fatores de risco da retinopatia da prematuridade no Hospital Universitário Onofre Lopes, Natal (RN) - Brasil
}

\author{
Incidence and risk factors of retinopathy of prematurity in University \\ Hospital Onofre Lopes, Natal (RN) - Brazil
}

\author{
Aline Macêdo Pinheiro ${ }^{1}$ \\ Wallace Andrino da Silva ${ }^{2}$ \\ Cíntia Glenda Freitas Bessa ${ }^{3}$ \\ Hélida Machado Cunha ${ }^{4}$ \\ Maria Ângela Fernandes Ferreira ${ }^{5}$ \\ Alexandre Henrique Bezerra Gomes ${ }^{6}$
}

\begin{tabular}{|c|}
\hline RESUMO \\
\hline 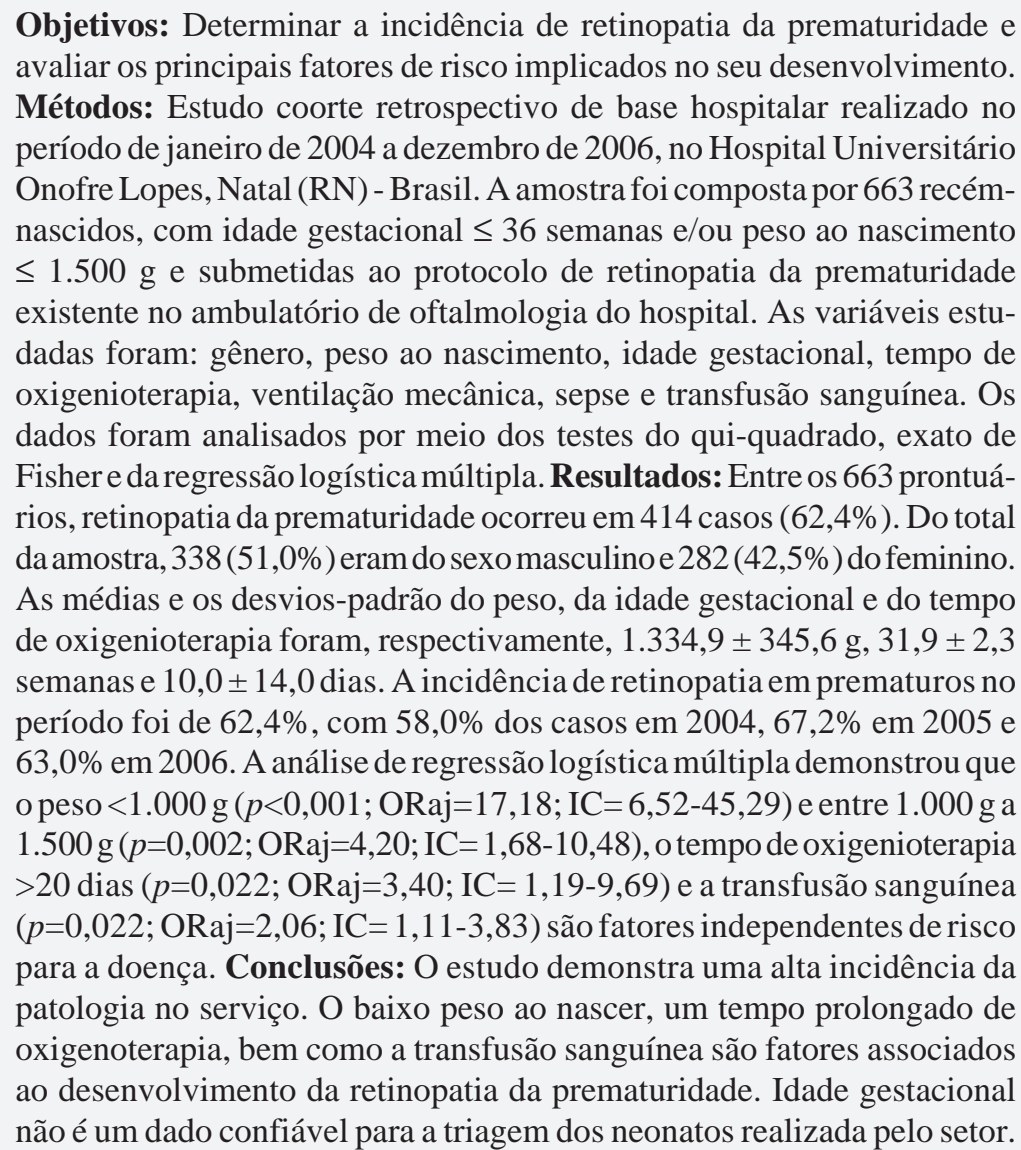 \\
\hline
\end{tabular}

Descritores: Retinopatia da prematuridade/epidemiologia; Fatores de risco; Recém-nascido, prematuro; Recém-nascido, de baixo peso

\section{INTRODUÇÃO}

A retinopatia da prematuridade (ROP) é uma doença vasoproliferativa da retina, de etiologia multifatorial ${ }^{(1)}$, relacionada principalmente à prematuridade, oxigenioterapia prolongada $^{(1-2)}$ e ao baixo peso ao nascer. Além desses, podemos citar outros fatores de risco, tais como: gemelaridade, sepse, transfusão sanguínea, hemorragia intraventricular, fototerapia, apnéia e anemia ${ }^{(1-2)}$. 
Apresenta uma fase inicial aguda e assintomática, na qual a vasculogênese normal é interrompida e a retina imatura apresenta uma transformação e proliferação celular. Tal fase pode evoluir para um processo fibrótico ou involuir espontaneamente, o que ocorre na maioria dos $\operatorname{casos}^{(1,3)}$.

Nos EUA, a retinopatia da prematuridade é a segunda causa mais comum de cegueira entre crianças com menos de 6 anos de idade e é estimado que de cada 100.000 crianças latino-americanas cegas, 24.000 os são em consequência da $\mathrm{ROP}^{(4)}$.

Atualmente, com o avanço das unidades de terapia intensiva neonatais, a sobrevida de recém-nascidos cada vez mais prematuros e com baixo peso vem aumentando e, por conseguinte, a ocorrência de ROP está se tornando cada vez maior ${ }^{(5)}$. Essa temática torna-se ainda mais importante em países com níveis intermediários de desenvolvimento humano, nos quais o número de unidades neonatais supera os programas de triagem e tratamento para $\mathrm{ROP}^{(5)}$.

No Brasil, a cegueira causada pela ROP adquiriu importância como problema de saúde pública a partir de um simpósio sobre ROP em 2002, que foi patrocinado através de uma parceria de organizações brasileiras de oftalmologia e pediatria e de organizações não-governamentais ${ }^{(6)}$.

O objetivo desse estudo é determinar a incidência de ROP no serviço de oftalmologia do Hospital Universitário Onofre Lopes (HUOL) e avaliar os principais fatores de risco implicados no seu desenvolvimento.

\section{MÉTODOS}

\section{Desenho do estudo}

Trata-se de um estudo coorte retrospectivo realizado a partir da análise de prontuários dos neonatos prematuros atendidos, no período de janeiro de 2004 a dezembro de 2006, no serviço de oftalmologia do HUOL, em Natal (RN) - Brasil.

O estudo foi aprovado pelo Comitê de Ética em Pesquisa do Hospital Universitário Onofre Lopes (CEP - HUOL), sob o protocolo 131/07.

\section{Cálculo e critérios de seleção da amostra}

O cálculo da amostra foi obtido a partir de um estudo piloto, onde se verificou uma probabilidade de exposição (peso $<1.500 \mathrm{~g}$ ) de $41,8 \%$ entre neonatos não acometidos por ROP. A partir dessa probabilidade e de uma odds ratio estimada em 2 , um nível de confiança de $99 \%$ e um poder de $80 \%$, determinou-se uma amostra mínima de 192 pacientes para cada grupo (com ROP e sem ROP).

Foram incluídos recém-nascidos com idade gestacional (IG) $\leq 36$ semanas e/ou peso ao nascimento $\leq 1.500 \mathrm{~g}$, submetidos ao protocolo de ROP existente no ambulatório de oftalmologia. Foram excluídos da amostra aqueles cujo preenchimento quanto à presença ou ausência da ROP estava ausente e os que não possuíam registros sobre IG e peso ao nascimento.

Foram selecionados 663 prontuários de acordo com os critérios de inclusão estabelecidos, sendo 414 com ROP e 249 sem ROP.

\section{Coleta e análise dos dados}

A extração dos dados foi realizada a partir das informações contidas nos protocolos de atendimento do setor de oftalmologia.

As variáveis coletadas foram: a presença ou não de ROP variável dependente - e gênero, peso ao nascimento, IG, ocorrência de sepse, recebimento de transfusão sanguínea, tempo de exposição ao oxigênio (capuz, pressão positiva de vias aéreas CPAP ou ventilação mecânica) e emprego de ventilação mecânica - variáveis independentes.

O preenchimento do protocolo foi realizado pelo médico oftalmologista na $4^{\underline{a}}$ a $6^{\underline{a}}$ semana de vida, conforme preconizado na literatura. $\mathrm{O}$ diagnóstico da ROP foi aferido mediante exame fundoscópico sob midríase (fenilefrina a 2,5\% e tropicamida a $0,5 \%$ ). As características da criança e da mãe foram obtidas através do prontuário do paciente cujo preenchimento ocorreu na unidade onde transcorreu seu nascimento. Nesta, a IG foi adquirida através de ultrassonografia (USG), data da última menstruação (DUM) ou pelo método de Capurro.

\section{Análise estatística}

A análise descritiva foi realizada através de números absolutos e porcentuais das variáveis qualitativas e medidas de tendência central - médias e seus, respectivos, desvios-padrão, das variáveis quantitativas.

$\mathrm{Na}$ análise univariada, o teste qui-quadrado e o teste exato de Fisher foram utilizados para determinar a associação entre as variáveis independentes e o desfecho (ROP). A magnitude do efeito dos fatores de risco sobre o desfecho foi expressa através da odds ratio (OR). Ficou determinado um nível de confiança de $95 \%$, sendo considerado significativo, portanto, os valores com $p<0,05$.

A análise do efeito independente das variáveis intervenientes sobre o desfecho (ROP) foi realizada através da regressão logística múltipla, utilizando-se a modelagem do tipo backward. Todas aquelas que apresentaram $\mathrm{p}<0,20$ no teste de associação foram incluídas no processo de modelagem. $\mathrm{O}$ ajuste do modelo foi determinado pelo teste de Hosmer - Lemeshow.

Para as análises estatísticas foi utilizado o programa "Statistic Package for Social Sciences (SPSS) for Windows", versão 13.0 .

\section{RESULTADOS}

A população estudada foi composta por 663 neonatos, sendo $338(51,0 \%)$ do sexo masculino. A média do peso ao nascimento foi igual a $1.334,9 \pm 345,6 \mathrm{~g}$. A idade gestacional variou de 23,0 a 39,6 semanas, sendo a média igual a 31,9 $\pm 2,3$ semanas. A média do tempo de oxigenioterapia foi igual a $10,0 \pm 14,0$ dias. Foram submetidos à ventilação mecânica 233 $(35,1 \%)$ pacientes; $304(45,9 \%)$ prematuros tiveram sepse neonatal e $185(27,9 \%)$ receberam transfusão sanguínea (Tabela 1).

A prevalência total de ROP no serviço, durante o período analisado, foi de $62,4 \%$, correspondendo a 414 casos. O grupo 
sem ROP foi composto por 249 pacientes (37,5\%). A distribuição de casos por ano se encontra demonstrada no gráfico 1 .

$\mathrm{Na}$ análise univariada, as variáveis - peso $\leq 1.000 \mathrm{~g}$, o peso de $1.000 \mathrm{~g}-1.500 \mathrm{~g}$, a IG $<28 \mathrm{~g}$, o tempo de oxigenioterapia de 11-20 dias e >20 dias, o uso de ventilação mecânica, a ocorrência de sepse e o emprego de transfusão sanguínea - apresentaram associação estatisticamente significativa $(p<0,05)$ com o desenvolvimento da ROP. Por outro lado, o gênero, a IG $\geq 34$ semanas e o tempo de oxigenioterapia $\leq 10$ dias não mostraram qualquer relação $(p>0,05)$ (Tabela 2$)$.

A análise de regressão logística múltipla demonstrou que o peso $<1.000 \mathrm{~g}(\mathrm{ORaj}=17,18 ; \mathrm{IC}=6,52-45,29)$ e entre $1.000 \mathrm{~g}$ a $1.500 \mathrm{~g}(\mathrm{ORaj}=4,20 ; \mathrm{IC}=1,68-10,48)$, o tempo de oxigenioterapia $>20$ dias ( $\mathrm{p}=0,022$; ORaj=3,40; IC=1,19-9,69) e a transfusão sanguínea (ORaj=2,06; IC=1,11-3,83) são fatores de risco independente para a ROP. O teste de Hosmer-Lemeshow apresentou valor de 0,716 . (Tabela 3 )

\section{DISCUSSÃO}

ROP continua sendo uma importante causa de cegueira prevenível no mundo ${ }^{(7)}$. Isso decorre, especialmente, do aumento nas taxas de sobrevidas de bebês muito prematuros com o desenvolvimento das unidades de terapia intensiva neonatais.

A incidência de ROP no nosso serviço se mostrou bastante alta, particularmente quando comparada com aquelas encontra-

\begin{tabular}{|lc|}
\hline \multicolumn{2}{|c|}{ Tabela 1. Características da população estudada } \\
Variável & Medidas descritivas \\
Gênero & \\
Masculino & $338,0(51,0 \%)$ \\
Feminino & $282,0(42,5 \%)$ \\
Peso ao nascimento (gramas) & $1.334,9( \pm 345,6)$ \\
Idade gestacional (semanas) & $31,9( \pm 2,3)$ \\
Tempo de oxigenioterapia (dias) & $10,0( \pm 14,0)$ \\
Ventilação mecânica & $233,0(35,1 \%)$ \\
Sepse & $304,0(45,9 \%)$ \\
Transfusão sanguínea & $185,0(27,9 \%)$ \\
\hline
\end{tabular}

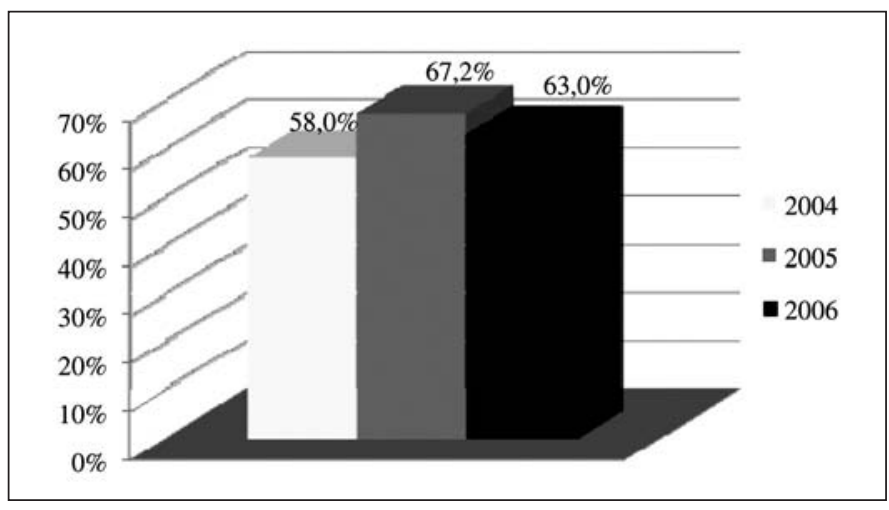

Gráfico 1 - Incidência anual de ROP no ambulatório de oftalmologia do HUOL, Natal (RN) - Brasil das na literatura. De uma maneira geral, os estudos demonstram uma prevalência que varia de $13,2 \%$ a $36,4 \%{ }^{(8-16)}$. Um estudo transversal realizado, em 2000, na Maternidade Escola Januário Cicco (MEJC), em Natal (RN), evidenciou uma incidência de $28 \%{ }^{(17)}$

Apenas a investigação realizada pelo estudo "Early Treatment for Retinopathy of Prematurity" (ETROP) ${ }^{(18)}$ detectou incidência de $68 \%$, semelhante aos nossos resultados. Vale ressaltar, no entanto, que sua amostra era constituída apenas por recém-nascidos de peso $<1.251 \mathrm{~g}$.

A alta frequência verificada em nosso estudo, muito provavelmente, se deve ao fato da amostra ter sido originada do setor de oftalmologia do HUOL, que é referência em atendimento de pré-termos da rede pública de saúde do Estado, o que permitiu maior detecção de casos.

Vale salientar ainda que o protocolo para ROP foi instaurado no hospital em 1998, o que permitiu uma padronização nos critérios diagnósticos e, consequentemente, uma maior confiabilidade nos dados obtidos.

Da mesma forma, a idade gestacional, a ventilação mecânica e a ocorrência de sepse não se mantiveram como fatores independentes de risco para a ocorrência de ROP após a análise multivariada. Alguns trabalhos na literatura estão em consonância com esse achado ${ }^{(6,15,19-20)}$, enquanto outros apontam a sepse como fator de risco ${ }^{(11,21-23)}$.

Embora diversos autores ${ }^{(9-10,13,16,19,21-24)}$ descrevam a influência da IG no desenvolvimento da ROP, no nosso estudo essa variável não se mostrou significativa quando submetida à análise multivariada, estando em conformidade com alguns relatos ${ }^{(11,15,20,25)}$. Isso, provavelmente, deve-se à baixa confiabilidade na determinação da idade gestacional em nosso Estado cuja definição se dá, em sua maioria, a partir da data da última menstruação (DUM) materna e/ou método de Capurro, os quais são bastante imprecisos. Em razão do baixo poder aquisitivo da população assistida, a USG do primeiro semestre, que possibilita medida mais acurada da IG, ainda é rara em nosso meio.

Em razão disso, o ponto de corte da IG para triagem, em nosso Estado, é de 35 semanas e não 32 semanas como preconizado no Brasil. Pois, a utilização de 32 semanas como ponto de corte poderia deixar passar prematuros que necessitem de avaliação oftalmológica.

Já no que se refere às variáveis, peso ao nascimento, tempo de oxigenioterapia e transfusão sanguínea, foi encontrada uma expressiva associação com o desenvolvimento de ROP.

Os dados deste estudo demonstraram que quanto menor o peso, maior a chance em desenvolver a doença, o que está de acordo com a maioria dos estudos que apontam para o muito baixo peso como fator de risco para aquisição da $\operatorname{ROP}^{(6,11,15,17,19-27)}$.

Tanto é que o peso $\leq 1.500 \mathrm{~g}$ corresponde a um dos critérios de avaliação para a doença e encontra-se presente nos principais "guidelines" elaborados por instituições internacionais (Academia Americana de Pediatria - AAP, Associação Americana para Oftalmologia Pediátrica e Estrabismo e Academia Americana de Oftalmologia) e nacionais (Sociedade Brasileira 


\begin{tabular}{|c|c|c|c|c|c|}
\hline \multirow[t]{2}{*}{ Variável } & \multicolumn{2}{|c|}{ ROP } & \multirow[t]{2}{*}{ ORbr } & \multirow[t]{2}{*}{ IC $95 \%$} & \multirow[t]{2}{*}{$p^{*}$} \\
\hline & Presença & Ausência & & & \\
\hline Gênero & $390(62,9 \%)$ & $230(37,1 \%)$ & 1,20 & $0,87-1,67$ & 0,270 \\
\hline \multicolumn{6}{|l|}{ Peso (gramas) } \\
\hline Menor que 1.000 & $96(91,4 \%)$ & $9(8,6 \%)$ & 19,17 & $9,07-40,02$ & $<0,001$ \\
\hline Entre 1.000 e 1.500 & $252(66,8 \%)$ & $125(33,2 \%)$ & 5,29 & $2,59-10,83$ & $<0,001$ \\
\hline Maior que 1.500 & $64(35,8 \%)$ & $115(64,2 \%)$ & 1,00 & - & - \\
\hline \multicolumn{6}{|c|}{ Idade gestacional (semanas) } \\
\hline Menor que 28 & 31 (93,9\%) & $2(6,1 \%)$ & 21,70 & $3,47-135,75$ & $<0,001^{* *}$ \\
\hline De 28 a 34 & $313(64,5 \%)$ & $172(35,5 \%)$ & 2,55 & $0,80-8,15$ & 0,129 \\
\hline De 34 a 36 & $54(45,0 \%)$ & $66(55,0 \%)$ & 1,14 & $0,34-3,81$ & 1,000 \\
\hline Maior que 36 & $5(41,7 \%)$ & $7(58,3 \%)$ & 1,00 & - & - \\
\hline Ventilação mecânica & $178(76,4 \%)$ & $55(23,6 \%)$ & 2,82 & $1,94-4,10$ & $<0,001$ \\
\hline \multicolumn{6}{|c|}{ Tempo de oxigenioterapia (dias) } \\
\hline $1-5$ & $111(46,6 \%)$ & $127(53,4 \%)$ & 1,00 & - & - \\
\hline $6-10$ & $93(69,4 \%)$ & $41(30,6 \%)$ & 2,49 & $0,95-6,56$ & 0,064 \\
\hline $11-20$ & $55(78,6 \%)$ & $15(21,4 \%)$ & 4,03 & $1,70-9,55$ & $<0,001$ \\
\hline Mais de 20 & $64(90,1 \%)$ & $7(9,9 \%)$ & 10,46 & $4,60-23,77$ & $<0,001$ \\
\hline Sepse & $227(74,7 \%)$ & $77(25,3 \%)$ & 3,07 & $2,14-4,39$ & $<0,001$ \\
\hline Transfusão sanguínea & $154(83,2 \%)$ & $31(16,8 \%)$ & 4,72 & $3,05-7,32$ & $<0,001$ \\
\hline
\end{tabular}

\begin{tabular}{|c|c|c|c|c|c|c|}
\hline Variável & ORbr & IC $95 \%$ & $p^{*}$ & ORaj & IC $95 \%$ & $p^{*}$ \\
\hline \multicolumn{7}{|l|}{ Peso (gramas) } \\
\hline Menor que 1.000 & 19,17 & $9,07-40,02$ & $<0,001$ & 17,18 & $6,52-45,29$ & $<0,001$ \\
\hline Entre 1.000 e 1.500 & 5,29 & $2,59-10,83$ & $<0,001$ & 4,20 & $1,68-10,48$ & 0,002 \\
\hline Maior que 1.500 & 1,00 & - & - & 1,00 & - & - \\
\hline \multicolumn{7}{|c|}{ Tempo de oxigenioterapia (dias) } \\
\hline $1-5$ & 1,00 & - & - & 1,00 & - & - \\
\hline $6-10$ & 2,49 & $0,95-6,56$ & 0,064 & 1,02 & $0,33-3,13$ & 0,971 \\
\hline $11-20$ & 4,03 & $1,70-9,55$ & $<0,001$ & 1,65 & $0,59-4,64$ & 0,340 \\
\hline Mais de 20 & 10,46 & $4,60-23,77$ & $<0,001$ & 3,40 & $1,19-9,69$ & 0,022 \\
\hline Transfusão sanguínea & 4,72 & $3,05-7,32$ & $<0,001$ & 2,06 & $1,11-3,83$ & 0,022 \\
\hline
\end{tabular}

de Pediatria, Conselho Brasileiro de Oftalmologia e Sociedade Brasileira de Oftalmologia Pediátrica).

O baixo peso como fator de risco sugere que fatores envolvidos no crescimento e desenvolvimento do indivíduo estejam implicados na etiologia dessa patologia. Trabalho feito em animais ${ }^{(26)}$ e fundamentado em achados clínicos aponta o fator-1 de crescimento insulina-símile (IGF-1), o qual já é sabidamente importante no crescimento e desenvolvimento fetal, como participante fundamental na formação vascular retiniana. $\mathrm{O}$ nível sérico de IGF-1 se correlaciona com o crescimento fetal. Com isso, uma expressão menor desse fator contribuiria para um feto com peso reduzido e, portanto, com maior risco para $\mathrm{ROP}^{(26)}$.

No que se refere ao tempo de oxigenioterapia, assim como apresentado em diversos outros resultados da literatura $^{(11,14-15,19-22,24,27)}$, é um importante fator a ser levado em consideração no rastreamento de casos.
Os níveis arteriais de oxigênio interferem na formação vascular retiniana do neonato. A terapia de suplementação com oxigênio, geralmente empregada em prematuros, expõe a retina à pressão arterial de oxigênio $\left(\mathrm{PaO}_{2}\right)$ variando de 60 a $100 \mathrm{mmHg}$. Normalmente, a vascularização retiniana se processa intraútero sob baixas pressões de oxigênio - $\mathrm{PaO}_{2}$ igual a $30 \mathrm{mmHg}$.

Numa primeira fase, a hiperóxia provoca obliteração dos vasos já formados e suprime a produção do fator de crescimento endotelial vascular (VEGF), comprometendo a angiogênese e causando hipóxia retiniana. Num segundo momento, essa hipóxia estimula um aumento do VEGF e, com isso, provoca uma neovascularização patológica. Ambas as alterações contribuem sobremaneira para o desenvolvimento da doença ${ }^{(26)}$.

O recebimento de transfusão sanguínea também expõe o prematuro ao desenvolvimento da doença. Alguns trabalhos atribuem esse risco às alterações hemodinâmicas durante a transfusão em bebês prematuros que têm volume sanguíneo 
reduzido. Além disso, as hemácias adultas, devido a sua menor afinidade pelo oxigênio, poderiam causar toxicidade tecidual pela maior liberação desse elemento no tecido retiniano ${ }^{(11,28)}$.

A avaliação das características dos recém-nascidos prematuros e de seus fatores de risco é fundamental para identificar o grupo de risco e, consequentemente, tornar mais eficaz sua triagem, possibilitando a prevenção da cegueira e reduzindo a incidência da ROP ao longo do tempo.

\section{CONCLUSÃO}

A alta incidência de ROP em infantes prematuros no HUOL e sua potencial causa de cegueiras servem de alerta para a importância do protocolo de triagem oftalmológica nas primeiras semanas de vida e para a necessidade de acompanhamento desse pacientes.

Prevenção da prematuridade e parcimônia nas condutas de uso de oxigênio e transfusão sanguínea em unidades de terapia intensiva neonatais poderão contribuir para a redução futura da incidência de ROP no serviço.

A insignificância da idade gestacional sinaliza para a necessidade de aprimoramento na obtenção dessa variável e para sua inutilidade como critério de triagem nos compreendidos ente 34 e 36 semanas. Sugerimos então 34 semanas como sendo o ponto de corte na triagem dos recém-nascidos se aproximando da realidade proposta pelo grupo ROP Brasil.

\section{AGRADECIMENTOS}

Agradecemos à Dra. Isa Maria Hetzel de Macedo, professora do Departamento de Saúde Coletiva e do Núcleo de Estudos em Saúde Coletiva (NESC) da UFRN e à Dra. Cláudia Rodrigues Souza Maia, professora do Departamento de Pediatria e Neonatologista da MEJC, pelas inestimáveis colaborações e disponibilidade em dividir seus conhecimentos para conosco.

\section{ABSTR ACT}

Purposes: To determine the incidence of retinopathy of prematurity and assess the main risk factors involved in its development. Methods: Retrospective cohort study carried out from January 2004 to December 2006, at University Hospital Onofre Lopes, Natal (RN) - Brazil. The sample was composed of 663 newborns, with less than or equal to 36 weeks of gestational age and/or birth weight less than or equal to $1,500 \mathrm{~g}$, submitted to the protocol of retinopathy of prematurity in the ophthalmology department of the hospital. The variables were: gender, birth weight, gestational age, duration of oxygen therapy, mechanical ventilation, sepsis and blood transfusion. Data were analyzed through the chi-squared test, Fisher's exact test and logistic regression model. Results: Of the 663 cases, retinopathy of prematurity occurred in 414 (62.4\%). Of the total sample, $338(51.0 \%)$ were male and 282 (42.5\%) female. Mean and standard deviation of weight, gestational age and duration of oxygen therapy were, respectively, $1,334.9 \pm 345.6 \mathrm{~g}$, $31.9 \pm 2.3$ weeks and $10.0 \pm 14.0$ days. The incidence of retinopathy in premature newborns in the period was $62.4 \%$, $58.0 \%$ of cases in 2004, $67.2 \%$ in 2005 and $63.0 \%$ in 2006. The multiple logistic regression analysis showed that the weight less than $1,000 \mathrm{~g}(\mathrm{p}<0.001 ; \operatorname{adjOR}=17.18, \mathrm{CI}=6.52-45.29)$ and between $1,000 \mathrm{~g}$ and $1,500 \mathrm{~g}(\mathrm{p}=0.002$; adjOR $-4.20 \mathrm{CI}=$ $1.68-10.48)$, the time of oxygen lounger than 20 days $(\mathrm{p}=0.022$; $\operatorname{adjOR}=3.40, \mathrm{CI}=1.19-9.69)$ and blood transfusion $(\mathrm{p}=0.022$; adjOR $-2.06, \mathrm{CI}=1.11-3.83)$ are independent factors for the disease. Conclusions: The study showed a high incidence of the disease in the service. A low birth weight, a prolonged oxygen therapy and blood transfusion are factors associated with the development of retinopathy of prematurity. Gestational age is not a reliable parameter for screening of newborns in the service.

Keywords: Retinopathy of prematurity/epidemiology; Risk factors; Newborn infant, premature; Newborn infant, low birth weight

\section{REFERÊNCIAS}

1. Moraes NSB, Graziano RM, Moreira Jr CA. Retinopatia da prematuridade. In: Abujamra S, Ávila M, Barsante C, Farah ME, Gonçalves JOR, Lavinsky J, et al. Retina e Vítreo: Clínica e Cirurgia. São Paulo: Roca; 2000. p.535-43.

2. Englert JA, Saunders RA, Purohit D, Hulsey TC, Ebeling M. The effect of anemia on retinopathy of prematurity in extremely low birth weight infants. $\mathrm{J}$ Perinatol. 2001;21(1):21-6.

3. Aggarwal R, Agarwal R, Deorari AK, Paul VK. Retinopathy of prematurity. Indian J Pediatr. 2002;69(1):83-6.

4. Gilbert C, Rahi J, Eckstein M, O’Sullivan J, Foster A. Retinopathy of prematurity in middle-income countries. Lancet. 1997;350(9070):12-4.

5. Quinn GE. Retinopathy of prematurity in Brazil: an emerging problem. J Pediatr (Rio J). 2007;83(3):191-3.

6. Fortes Filho JB, Eckert GU, Procianoy L, Barros CK, Procianoy RS. Incidence and risk factors for retinopathy of prematurity in very low and in extremely low birth weight infants in a unit-based approach in southern Brazil. Eye. 2009; 23(1):25-30.

7. Clemett R, Darlow B. Results of screening low-birth-weight infants for retinopathy of prematurity. Curr Opin Ophthalmol. 1999;10(3):155-63.

8. Rodriguez-Hurtado FJ, Canizares JM. [Screening for retinopathy of prematurity. Our experience about limits of birth weight, post-conceptional age and other risk factors]. Arch Soc Esp Oftalmol. 2006;81(5):275-9. Spanish.

9. Sluncheva B, Vakrilova L, Emilova Z, Doicheva E, Topov A. [Retinopathy of prematurity - frequency and risk factors]. Akush Ginekol (Sofiia). 2002;42(1): 10-4. Bulgarian.

10. Glowacka E, Kwinta P, Mitkowska Z, Kobylarz J, Kaminska-Mroczkowska H, Mazurek M, et al. [Risk factors of retinopathy of prematurity in newborns treated in neonatal intensive care unit of University Children's Hospital Collegium Medicum Jagiellonian University]. Przegl Lek. 2002;59(Suppl 1): 86-90. Polish.

11. Gupta VP, Dhaliwal U, Sharma R, Gupta P, Rohatgi J. Retinopathy of prematurity - risk factors. Indian J Pediatr. 2004;71(10):887-92.

12. Sharma R, Gupta VP, Dhaliwal U, Gupta P. Screening for retinopathy of prematurity in developing countries. J Trop Pediatr. 2007;53(1):52-4.

13. Lermann VL, Fortes Filho JB, Procianoy RS. The prevalence of retinopathy of prematurity in very low birth weight newborn infants. J Pediatr (Rio J). 2006; 82(1):27-32.

14. Graziano RM, Leone CR, Cunha SL, Pinheiro AC. Prevalence of retinopathy of prematurity in very low birth weight infants. J Pediatr (Rio J).1997;73(6):377-82.

15. Shah VA, Yeo CL, Ling YL, Ho LY. Incidence, risk factors of retinopathy of 
prematurity among very low birth weight infants in Singapore. Ann Acad Med Singapore. 2005;34(2):169-78.

16. Larsson E, Carle-Petrelius B, Cernerud G, Ots L, Wallin A, Holmström G. Incidence of ROP in two consecutive Swedish population based studies. Br J Ophthalmol. 2002;86(10):1122-6. Comment in: Br J Ophthalmol. 2002;86(10): 1071.

17. Garcia CA, Gomes AH, Barbosa MF, Rocha ML, Uchôa RA. Avaliação oftalmológica e fatores de risco da retinopatia da prematuridade na Maternidade Escola Januário Cicco, Natal - RN. Rev Bras Oftalmol. 2001;60(7):489-95.

18. Good WV, Hardy RJ, Dobson V, Palmer EA, Phelps DL, Quintos M, Tung B Early Treatment for Retinopathy of Prematurity Cooperative Group. The incidence and course of retinopathy of prematurity: findings from the early treatment for retinopathy of prematurity study. Pediatrics. 2005;116(1):15-23.

19. Lala-Gitteau E, Majzoub S, Saliba E, Pisella PJ. [Epidemiology for retinopathy of prematurity: risk factors in the Tours hospital (France)]. J Fr Ophtalmol. 2007;30(4):366-73.

20. Al-Amro SA, Al-Kharfi TM, Thabit AA, Al-Mofada SM. Risk factors for acute retinopathy of prematurity. Compr Ther. 2007;33(2):73-7.
21. Bonotto LB, Moreira AT, Carvalho DS. [Prevalence of retinopathy of prematurity in premature babies examined during the period 1992-1999, Joinville (SC): evaluation of associated risks - screening]. Arq Bras Oftalmol. 2007;70(1):55-61. Portuguese.

22. Ahmed AS, Muslima H, Anwar KS, Khan NZ, Chowdhury MA, Saha SK, et al. Retinopathy of prematurity in Bangladeshi neonates. J Trop Pediatr. 2008 54(5):333-9.

23. Bassiouny MR. Risk factor associated with retinopathy of prematurity: a study from Oman. J Trop Pediatr. 1996;42(6):355-8.

24. Seiberth V, Linderkamp O. Risk factors in retinopathy of prematurity. A multivariate statistical analysis. Ophthalmologica. 2000;214(2):131-5.

25. Lad EM, Nguyen TC, Morton JM, Moshfeghi DM. Retinopathy of prematurity in the United States. Br J Ophthalmol. 2008;92(3):320-5.

26. Chen J, Smith LE. Retinopathy of prematurity. Angiogenesis. 2007;10(2):133-40

27. Karna P, Muttineni J, Angell L, Karmaus W. Retinopathy of prematurity and risk factors: a prospective cohort study. BMC Pediatr. 2005;5(1):18

28. Chawla D, Agarwal R, Deorari AK, Paul VK. Retinopathy of prematurity. India J Pediatr. 2008;75(1):73-6. 